\title{
Empirically derived dietary patterns in relation to psychological disorders
}

\author{
Mahdieh Hosseinzadeh', Mohammadreza Vafa' ${ }^{2}$, Ahmad Esmaillzadeh ${ }^{3,4, *}$, \\ Awat Feizi ${ }^{5}$, Reza Majdzadeh ${ }^{6}$, Hamidreza Afshar ${ }^{7}$, Ammar Hassanzadeh Keshteli ${ }^{8,9}$ and \\ Peyman Adibi ${ }^{9}$ \\ 'Department of Community Nutrition, School of Nutritional Sciences and Dietetics, Tehran University of Medical \\ Sciences, Tehran, Islamic Republic of Iran: ${ }^{2}$ Department of Nutrition, Iran University of Medical Sciences, Tehran, \\ Islamic Republic of Iran: ${ }^{3}$ Food Security Research Center, Isfahan University of Medical Sciences, Isfahan, Islamic \\ Republic of Iran: ${ }^{4}$ Department of Community Nutrition, School of Nutrition and Food Science, Isfahan University of \\ Medical Sciences, PO Box 81745-151, Isfahan, Islamic Republic of Iran: ${ }^{5}$ Department of Epidemiology and \\ Biostatistics, School of Public Health, Isfahan University of Medical Sciences, Isfahan, Islamic Republic of Iran: \\ ${ }^{6}$ Department of Epidemiology and Biostatistics, School of Public Health and Knowledge Utilization Research Center, \\ Tehran University of Medical Sciences, Tehran, Islamic Republic of Iran: ${ }^{7}$ Psychosomatic Research Center, Isfahan \\ University of Medical Sciences, Isfahan, Islamic Republic of Iran: ${ }^{8}$ Department of Medicine, University of Alberta, \\ Edmonton, Alberta, Canada: ${ }^{9}$ Integrative Functional Gastroenterology Research Center, Isfahan University of \\ Medical Sciences, Isfahan, Islamic Republic of Iran
}

Submitted 21 October 2014: Final revision received 3 March 2015: Accepted 21 April 2015: First published online 10 June 2015

\begin{abstract}
Objective: Psychological disorders are highly prevalent worldwide. The present study aimed to investigate the relationship between major dietary patterns and prevalence of psychological disorders in a large sample of Iranian adults.

Design: A cross-sectional study was done to identify dietary patterns derived from factor analysis. Dietary data were collected through the use of a validated dishbased semi-quantitative FFQ. Psychological health was examined by use of the Hospital Anxiety and Depression Scale and the General Health Questionnaire. Setting: The study was conducted in Isfahan, Iran, within the framework of the Study on Epidemiology of Psychological, Alimentary Health and Nutrition (SEPAHAN).

Subjects: Iranian adults ( $n$ 3846) aged $20-55$ years.

Results: After adjustment for potential confounders, greater adherence to the lactovegetarian dietary pattern was protectively associated with depression in women $(\mathrm{OR}=0.65 ; 95 \%$ CI $0.46,0.91)$. Normal-weight participants in the top quintile of this dietary pattern tended to have decreased odds of anxiety compared with those in the bottom quintile $(\mathrm{OR}=0.61 ; 95 \% \mathrm{CI} 0.38,1 \cdot 00)$. In addition, the traditional dietary pattern was associated with increased odds of depression (OR $=1.42 ; 95 \%$ CI 1.01, 1.99) and anxiety (OR=1.56; $95 \%$ CI 1.00, 2.42) in women. Normalweight participants in the highest quintile of the traditional dietary pattern had greater odds for anxiety (OR $=1.89$; $95 \%$ CI 1.12, 3.08) compared with those in the lowest quintile. The Western dietary pattern was associated with increased odds of depression in men $(\mathrm{OR}=1.73 ; 95 \% \mathrm{CI} 1 \cdot 07,2 \cdot 81)$ and anxiety in normal-weight participants $(\mathrm{OR}=2.05 ; 95 \% \mathrm{CI} 1.22,3.46)$. There was a significant increasing trend in the odds of psychological distress across increasing quintiles of the fast food dietary pattern in women $(P$-trend $=0 \cdot 02)$.

Conclusions: Recommendation to increase the intake of fruits, citrus fruits, vegetables, tomato and low-fat dairy products and to reduce the intakes of snacks, high-fat dairy products, chocolate, carbonated drinks, sweets and desserts might be associated with lower chance of psychological disorders.
\end{abstract}


Earlier studies investigating diet-disease relationships have mostly focused on isolated nutrients or foods; however, nutrients or foods are consumed together. Thus a single nutrient or food cannot completely explain the aetiology of a chronic condition ${ }^{(1)}$. Dietary pattern approach, as a new direction in nutritional epidemiology, has recently emerged to take the combined effects of nutrients and foods into account ${ }^{(2,3)}$. This approach can provide a comprehensive picture of food and nutrient interactions and can be efficiently applied in the community setting to reduce chronic diseases ${ }^{(4)}$. Data-driven and hypothesis-oriented methods have been used to identify dietary patterns ${ }^{(5)}$.

Psychological disorders including depression and anxiety are highly prevalent worldwide ${ }^{(6,7)}$. Depression is the fourth leading cause of disease burden and the main cause of disability worldwide ${ }^{(6,7)}$. Although not a prominent cause of mortality, depression results in significantly decreased quality of life ${ }^{(8)}$. Previous studies on diet and psychological disorders have assessed the association between dietary intakes of folate, vitamin $\mathrm{B}_{6}$, vitamin $\mathrm{B}_{12}$, long-chain fatty acids, $\mathrm{Zn}$ and $\mathrm{Mg}$ and depression ${ }^{(9-15)}$. Limited data are available linking dietary patterns to psychological disorders. In a study on Australian adult women, consumption of a 'traditional' dietary pattern containing high amounts of vegetables, fruit, meat, fish and whole grains was associated with lower odds of depression (16). Adherence to a 'processed food' dietary pattern was linked with increased risk, while a 'whole food' dietary pattern decreased the risk of depression in British middle-aged women ${ }^{(17)}$. Similar findings have also been reported from Norway ${ }^{(18)}$ as well as from Chinese adolescents $^{(19)}$.

Almost all previous reports on dietary patterns and depression came from Western populations and we are not aware of any report in non-Western nations, particularly in Middle Eastern populations, where the dietary intakes are highly different from those in other parts of the world ${ }^{(20)}$. For instance, studies have estimated the prevalence of psychiatric disorders in the Iranian population as $10.81 \%$; more common in females (14.34\%) than males (7.34\%). The prevalence of anxiety and mood disorders was $8.35 \%$ and $4.29 \%$, respectively ${ }^{(21)}$. This is higher than reported in China ${ }^{(22)}$ and Japan ${ }^{(23)}$, and lower than that reported in the $\mathrm{USA}^{(24)}$. Therefore, the association between dietary patterns and psychological disorders in this part of the world might provide some novel insights into diet-disease relationships. Given the high prevalence of depression in this area ${ }^{(8,21)}$, it seems that dietary patterns might play a key role. In addition, earlier studies have mostly focused on depression, and the association between dietary intakes and other psychological disorders like anxiety and psychological distress has not been adequately addressed. Due to the contribution of several nutrients and foods to these conditions ${ }^{(9-15)}$, it is expected to find a significant association between major dietary patterns and these disorders. Moreover, previous studies from this region have indicated that the application of statistical methods like factor analysis on dietary data would result in interpretable dietary patterns ${ }^{(20,25,26)}$; however, almost all previous reports on dietary patterns from this region have been conducted on small sample sizes. For example, dietary patterns have been identified on a sample of 486 female subjects ${ }^{(20,25)}$ and 150 participants ${ }^{(26)}$ in different studies from the region. However, it remains unknown if the application of these methods on a representative large sample could provide meaningful dietary patterns.

Therefore the present study was done to examine the relationship between major dietary patterns derived from factor analysis and prevalence of psychological disorders in a large sample of Iranian adults.

\section{Participants and methods}

\section{Study population}

The current study was conducted within the framework of the Study on the Epidemiology of Psychological, Alimentary Health and Nutrition (SEPAHAN), a cross-sectional study that aimed to examine the epidemiological concepts of functional gastrointestinal disorders (FGID) and their association with lifestyle and psychological determinants. Detailed information about the study design, sampling procedures, participants' characteristics and data collection process has been published previously ${ }^{(27)}$. Briefly, the SEPAHAN project was designed based on FGID as its main outcome of interest. Therefore, the sample size calculation was based on this variable. We hypothesized that the prevalence of FGID in Iran would be $15 \%$. We further hypothesized that psychological disorders or eating a diet low in fibre would double the risk of having any FGID. With an $80 \%$ power, a type I error of 0.05 and desired confidence interval of 0.03 , the minimum required sample size was calculated to be 1387 subjects. As mentioned, this sample size was calculated for FGID as the main outcome of the SEPAHAN study. Because the prevalence of psychological disorders is lower than that of FGID in the Iranian population, the required sample size for assessing psychological disorders as the main outcome in the current study would even be lower than 1387 people. Therefore, 3846 participants recruited in the current analysis seem to be enough.

We enrolled a sample of the Iranian adult population aged 20-55 years who were working in health centres. Isfahan University of Medical Sciences (IUMS) central office has direct contact with all staff in different cities and centres through fifty staff members working in Public Relations Units (PRU). Monthly sessions with PRU staff started four months prior to the recruitment of participants. In these sessions, the principal investigator of the project (P.A.) and its coordinator (A.H.K.) described the rationale 
and methodological aspects of SEPAHAN completely and answered PRU staff questions. Two months prior to the initiation of SEPAHAN, the first official letter was sent to all managers of IUMS units working in different cities and centres and the study was introduced to them briefly. Some of them later called the coordinator of the study and, if requested, more details were provided. The last letter was sent to the managers of IUMS units one week prior to the launch of the study. Forty-five days before distributing the first wave of questionnaires, staff in the selected centres were informed about the study through brochures and posters that were distributed among them by PRU staff. PRU staff informed participants about the contents of the questionnaires and study aims while they were handing out the questionnaires. Each participant was provided with an envelope in which to put the completed questionnaires and asked to return the completed questionnaires to PRU staff within $7 \mathrm{~d}$. All questionnaires were distributed and collected within three weeks and sent to the main office of the project.

Data collection and data entry were monitored continuously by the principal investigator of the project (P.A.) and its coordinator (A.H.K.). We collected data in two separate phases with a short period (3-4 weeks) between them to increase the accuracy of data collection as well as the response rate. In the first phase, all participants were asked to complete a self-administered questionnaire on demographic and lifestyle factors including nutritional habits and dietary intakes (response rate: 86.16\%). In order to collect information on psychological health, another set of self-administered questionnaires was applied in the second phase. After linking data from both phases, 4763 adults who had complete information on both dietary data and psychological health were available for analysis. We excluded those who reported energy intake outside the range 3347-17573 kJ (800-4200 kcal). These exclusions left 3846 persons for the current analysis.

The IUMS Ethics Committee as well as the Tehran University of Medical Sciences Ethics Committee reviewed the study aims and procedures and then approved the study ethically for conduct.

\section{Dietary assessment}

Dietary data were collected using a self-administered, Willett-format, dish-based, 106-item semi-quantitative FFFQ (DS-FFQ), which was designed and validated specifically for Iranian adults ${ }^{(28)}$. Detailed information about the design, foods included as well as the validity of this questionnaire has been reported elsewhere. Briefly, the questionnaire contained five categories of foods and dishes: (i) mixed dishes (cooked or canned, twenty-nine items); (ii) grains (different types of bread, cakes, biscuits and potato, ten items); (iii) dairy products (dairy, butter and cream, nine items); (iv) fruits and vegetables (twentytwo items); and (v) miscellaneous food items and beverages (including sweets, fast foods, nuts, desserts and beverages, thirty-six items). To develop the questionnaire, a comprehensive list of foods and dishes commonly consumed by Iranian adults was constructed. Then, we chose those foods that were nutrient-rich, consumed reasonably often or contributed to between-person variation. This process led to the remaining of the 106 food items in the questionnaire. The portion size for food items and mixed dishes was defined based on the most commonly consumed portion size for each item in the general population. To increase precision and accuracy of estimates, we attempted to give the portion size of foods and mixed dishes as a unit with the same perception for all people. Participants were asked to report their dietary intakes of foods and mixed dishes based on nine multiplechoice frequency response categories varying from 'never or less than once a month' to 'twelve or more times per day'. The number of frequency response categories was not constant for all foods. For foods consumed infrequently, we omitted the high-frequency categories, while for common foods with a high consumption, the number of multiple choice-categories increased. The number of response categories for the food list varied from six to nine choices. For instance, the frequency response for tuna consumption included six categories, as follows: never or $<1$ time/month, 1-3 times/month, 1 time/week, 2-4 times/week, 5-6 times/week and 1-2 times/d; and for tea consumption the frequency response included nine categories, as follows: never or $<1$ cup/month, 1-3 cups/ month, 1-3 cups/week, 4-6 cups/week, 1 cup/d, 2-4 cups/d, 5-7 cups/d, 8-11 cups/d and $\geq 12 \mathrm{cups} / \mathrm{d}$. Finally, we computed daily intake of all food items and then converted to grams per day using household measures. Daily nutrient intakes for each participant were calculated using the US Department of Agriculture's national nutrient databank. To identify dietary patterns, we assigned each food item to one of thirty-nine predefined food groups (Table 1). Food items were included in a certain food group or as a distinct group based on the similarity of nutrients and their association with psychological health.

\section{Assessment of psychological bealth}

The Iranian validated version of the Hospital Anxiety and Depression Scale (HADS) was used to screen for anxiety and depression ${ }^{(29)}$. HADS is a brief and useful questionnaire to measure psychological disorders and assess the symptom severity of anxiety disorders and depression. The HADS contains fourteen items and consists of two subscales: anxiety and depression. Each item includes a four-point scale; higher scores indicate an elevated level of anxious and depressive symptomatology. The maximum score is 21 for anxiety and depression. Scores of $\geq 8$ on either subscale were considered as psychological disorders and scores of $0-7$ were defined as 'normal' in the current study ${ }^{(29)}$. 
Table 1 Food grouping used in the dietary pattern analyses

\begin{tabular}{ll}
\hline Food groups & Food items \\
\hline Meat & Meat, cooked meat \\
Processed meat & Sausage \\
Organ meats & Heart, liver and kidney, intestine and \\
& viscera \\
Fish & Fish \\
Poultry & Chicken \\
Eggs & Eggs \\
Butter & Butter \\
Low-fat dairy products & Dough, yoghurt, curd, milk, cheese \\
High-fat dairy products & Cream, ice cream, cheese pizza \\
Tea & Tea \\
Coffee & Coffee and espresso \\
Fruit & Apples, cherries, apricots, plums, \\
& fresh figs, kiwi, strawberries, \\
& grapes, fresh berries, dates, \\
& barberries, bananas, \\
Citrus & pomegranates \\
Fruit juices & Citrus \\
Onions & Lemon juice, juice \\
Non-flatulent vegetables & Onions, fried onions \\
& Mushrooms, carrots, vegetable, green \\
Flatulent vegetables & beans, herbs, lettuce, aubergines \\
Legumes & Cucumber, cabbage, peas \\
& Cereals, cotyledon, beans, vetch, \\
Whole grains & green peas, lentils \\
Refined grains & Sangak, barbari, diet bread, wheat \\
Snacks & Lavash, baguette bread, rice, flour, \\
Nuts & noodle, macaroni, biscuit \\
Mayonnaise & Chips \\
Dried fruit & Walnut, nuts \\
Sweets and desserts & Mayonnaise \\
Chocolate & Raisins, dried berries, limes \\
Hydrogenated fats & Gushfyl, pastry, cake \\
Vegetable oils & Types of chocolate \\
Sugars & Tail \\
Condiments & Oil \\
Tomatoes & Candy, sugar, tamarisk, dried sugar \\
Carbonated drinks & Jam, honey \\
Pickles & Tomatoes, tomato paste, red sauce \\
French fries & Drink \\
Salt & Pickles \\
Pepper & French fries \\
Cocoa & Salt \\
Potato & Pepper \\
Soya & Bakea potato \\
\hline & \\
\hline
\end{tabular}

The Iranian validated version of the General Health Questionnaire (GHQ) with twelve items was used to assess psychological distress ${ }^{(30)}$. GHQ-12 is a brief, simple, easy-to-complete instrument for measuring current and primary mental health that asks the respondent whether he/she has experienced a particular symptom of psychological distress recently. Each item consists of a four-point scale ('less than usual', 'no more than usual', 'rather more than usual', 'much more than usual'). There are two most common scoring methods, bimodal (0-0-1-1) and Likert scoring (0-1-2-3), giving a total score of 12 or 36 on the basis of the scoring method selected. We used the bimodal scoring style for the present study. This gives scores ranging from 0 to 12 . Higher scores indicate a greater degree of psychological distress. In the current study, psychological distress was defined as having the score of $\geq 5^{(30)}$.

\section{Assessment of covariates}

Required information on other variables including age, sex, marital status, socio-economic status (SES), smoking status, chronic conditions (diabetes, asthma, colitis, stroke, heart failure and cancers) and the use of antidepressant medications was obtained from demographic and medical history questionnaires. SES score was computed as an index of socio-economic status based on family size $(\leq 4$, $>4$ people), education (academic and non-academic education) and acquisition (house ownership or not). For each variable of the SES score, participants were given a score of 1 if they had $\leq 4$ family members, were academically educated or owned a house, and were given a score of 0 if they had $>4$ family members, had nonacademic education or had leasehold house. Then, total SES score was calculated by summing up the assigned scores (minimum SES score of 0 to maximum score of 3 ). Individuals with the score of 3 were considered as having high SES. Physical activity was assessed using the General Practice Physical Activity Questionnaire (GPPAQ) ${ }^{(31)}$ and those with more than $1 \mathrm{~h}$ of activity per week were considered as physically active. Anthropometric measures including weight, height and waist circumference were assessed using a self-administered questionnaire. BMI was calculated by dividing weight (in kilograms) by the square of height (in metres).

\section{Statistical analysis}

To identify major dietary patterns based on the thirty-nine food groups, we used principal component analysis and the factors were rotated by varimax rotation. The natural interpretation of the factors in conjunction with eigenvalues $>1.5$ and the scree plot determined whether a factor should be retained. The derived factors (dietary patterns) were labelled on the basis of our interpretation of the data and of the earlier literature. The factor score for each pattern was calculated by summing intakes of food groups weighted by their factor loadings, and each participant received a factor score for each identified pattern. We categorized participants by quintiles of dietary pattern scores. One-way ANOVA was used to examine significant differences in continuous variables across quintile categories of dietary pattern scores. The distribution of participants in terms of categorical variables across quintiles was assessed by means of the $\chi^{2}$ test. Age- and energyadjusted intakes of foods and nutrients across quintiles of dietary pattern scores were examined using ANCOVA. To find the association between dietary patterns and psychological disorders, we used logistic regression in different models. First, we controlled for age (continuous) and then for sex (categorical), marital status (married, single, widowed, divorced), education (under diploma, diploma, above diploma, bachelors and above), physical activity (never, <1 h/week, >1 h/week), chronic diseases (hypertension, diabetes, stroke, CVD, cancers), smoking 
(non-smoker, ex-smoker, current smoker), antidepressant use (yes, no) and energy intake (kcal/d). Further adjustments for BMI were done in the last model. Stratified analyses by sex and BMI status $\left(<25.0\right.$ and $\left.\geq 25 \cdot 0 \mathrm{~kg} / \mathrm{m}^{2}\right)$ were also done in age-adjusted and multivariable-adjusted models. The covariates in these models were the same as above. The potential confounders we adjusted for in the analyses were determined based on earlier publications that had examined the relationship of dietary patterns with psychological disorders ${ }^{(16-19)}$. In all analyses, the first quintile of dietary pattern scores was considered as a reference. To assess the overall trend of odds ratios across increasing quintiles of dietary pattern scores, we treated the quintile categories as an ordinal variable in the analyses. All analyses were performed using the statistical software package IBM SPSS Statistics version 19.0. $P$ values were considered significant at $<0 \cdot 05$.

\section{Results}

\section{Characteristics of the study population}

The mean age of the study population was 36.4 (SD 8.0) years. Prevalence of depression, anxiety and psychological distress was $10 \cdot 6 \%$ (men, $7 \cdot 0 \%$; women, $13.5 \%$ ), $5.7 \%$ (men, $4.4 \%$; women, $6.8 \%$ ) and $23.3 \%$ (men, $18.4 \%$; women, $26.5 \%$ ), respectively.

We identified four major dietary patterns: (i) a 'fast food' dietary pattern that was high in French fries, vegetable oils, meat, pepper, salt, onions, soya and egg; (ii) a 'traditional' dietary pattern that was high loaded by vegetable oils, meat, salt, legumes, non-flatulent vegetables, poultry, hydrogenated vegetable oils, dried fruits, fish and organ meats; (iii) a 'lacto-vegetarian' dietary pattern which was comprised mainly of non-flatulent vegetables, tomato, citrus fruits, flatulent vegetables, fruits and low-fat dairy products; and (iv) a 'Western' dietary pattern that was characterized by high intakes of snacks, high-fat dairy products, chocolate, carbonated drinks, sweets and desserts (Table 2 ). These dietary patterns explained $30 \cdot 2 \%$ of the whole variance in dietary intakes.

Characteristics of the study participants across quintiles of dietary pattern scores are provided in Table 3. Mean weight and BMI were not significantly different across quintile categories of different dietary pattern scores. We did not find any significant difference in other general characteristics of participants across quintile categories of the fast food and Western dietary pattern scores. Participants in the third quintile of the traditional dietary pattern score were older than those in the lowest quintile. Participants in the highest quintile of the lacto-vegetarian dietary pattern were slightly older and less likely to consume fried foods. No further overall significant difference was found across quintiles of dietary pattern scores.

Age- and energy-adjusted intakes of food groups and nutrients across quintile categories of dietary pattern scores
Table 2 Factor loading matrix for the major dietary patterns identified among the sample of Iranian adults ( $n$ 3846) aged 20-55 years

\begin{tabular}{|c|c|c|c|c|}
\hline \multirow[b]{2}{*}{ Food group } & \multicolumn{4}{|c|}{ Dietary pattern } \\
\hline & $\begin{array}{l}\text { Fast food } \\
\text { dietary } \\
\text { pattern }\end{array}$ & $\begin{array}{l}\text { Traditional } \\
\text { dietary } \\
\text { pattern }\end{array}$ & $\begin{array}{l}\text { Lacto- } \\
\text { vegetarian } \\
\text { dietary } \\
\text { pattern }\end{array}$ & $\begin{array}{c}\text { Western } \\
\text { dietary } \\
\text { pattern }\end{array}$ \\
\hline French fries & 0.84 & - & - & - \\
\hline Vegetable oils & 0.78 & 0.43 & - & - \\
\hline Meat & 0.72 & 0.45 & - & - \\
\hline Pepper & 0.71 & - & - & - \\
\hline Salt & 0.60 & 0.60 & - & - \\
\hline Onions & 0.56 & 0.20 & 0.26 & - \\
\hline Soya & 0.51 & - & - & - \\
\hline Egg & 0.47 & - & - & - \\
\hline Refined grains & 0.37 & - & - & - \\
\hline Legumes & 0.33 & 0.59 & - & - \\
\hline $\begin{array}{r}\text { Non-flatulent } \\
\text { vegetables }\end{array}$ & 0.29 & 0.44 & 0.52 & - \\
\hline Tomato & 0.22 & - & 0.54 & - \\
\hline Potato & 0.21 & 0.20 & - & - \\
\hline Poultry & - & 0.50 & - & - \\
\hline Tea & - & - & - & - \\
\hline Coffee & - & - & - & 0.20 \\
\hline Sugars & - & - & - & 0.32 \\
\hline $\begin{array}{l}\text { Hydrogenated } \\
\text { vegetable } \\
\text { oils }\end{array}$ & - & 0.51 & - & - \\
\hline Dried fruits & - & 0.43 & 0.32 & - \\
\hline Pickles & - & - & 0.20 & 0.34 \\
\hline Citrus fruits & - & - & 0.61 & - \\
\hline Whole grains & - & - & - & - \\
\hline $\begin{array}{l}\text { Flatulent } \\
\text { vegetables }\end{array}$ & - & - & $0 \cdot 61$ & - \\
\hline Mayonnaise & - & - & - & 0.34 \\
\hline $\begin{array}{l}\text { Processed } \\
\text { meats }\end{array}$ & - & 0.29 & -0.20 & 0.38 \\
\hline Fruits & - & - & 0.64 & - \\
\hline $\begin{array}{l}\text { Low-fat dairy } \\
\text { products }\end{array}$ & - & - & 0.41 & - \\
\hline $\begin{array}{l}\text { Carbonated } \\
\text { drinks }\end{array}$ & - & - & - & 0.42 \\
\hline $\begin{array}{c}\text { Sweets and } \\
\text { desserts }\end{array}$ & - & - & - & 0.53 \\
\hline Fish & - & 0.50 & - & - \\
\hline Butter & - & - & - & 0.28 \\
\hline Chocolate & - & - & - & 0.46 \\
\hline Nuts & - & - & 0.27 & 0.28 \\
\hline $\begin{array}{l}\text { High-fat dairy } \\
\text { products }\end{array}$ & - & - & - & 0.43 \\
\hline Fruit juice & - & 0.30 & 0.26 & 0.24 \\
\hline Condiments & - & - & - & - \\
\hline Organ meats & - & 0.50 & - & 0.20 \\
\hline Snacks & - & - & - & 0.46 \\
\hline Cacao milk & - & - & - & 0.26 \\
\hline $\begin{array}{l}\% \text { of variance } \\
\text { explained }\end{array}$ & $10 \cdot 5$ & $7 \cdot 7$ & $6 \cdot 3$ & $5 \cdot 6$ \\
\hline
\end{tabular}

Only items with correlation coefficients $\geq 0.20$ are presented.

are indicated in Table 4. Compared with those in the lowest quintile of the fast food dietary pattern, individuals in the top quintile had significantly higher intakes of vegetable oils, processed meat, egg, low-fat dairy products, vegetables, legumes, refined grains, French fries and pickles; however, they had lower intakes of coffee, fruits, whole grains and nuts. Adherence to the traditional dietary pattern 
was associated with higher intakes of vegetable oils, processed meat, egg, high fat dairy products, coffee, fruits, vegetables, legumes, whole grains, nuts, French fries and pickles, and lower consumption of refined grains, low-fat dairy products and chocolate. Participants in the top quintile of the lacto-vegetarian dietary pattern had significantly higher intakes of vegetable oils, low- and high-fat dairy products, coffee, fruits, vegetables, legumes, whole grains, nuts, chocolate and pickles, and lower intakes of meat and refined grains than those in the bottom quintile. High consumption of the Western dietary pattern was associated with higher intakes of vegetable oils, processed meat, egg, low- and high-fat dairy products, coffee, fruits, vegetables, whole and refined grains, nuts, chocolate, French fries and pickles, and lower intakes of legumes. Dietary intakes of energy, protein, fat, fibre vitamin C, cholesterol, trans-fatty acids, SFA, MUFA and PUFA were significantly higher among those in the top quintile, than among those in the lowest, of the fast food, traditional and Western dietary patterns. There was also a significant difference across quintiles of the lacto-vegetarian dietary pattern in terms of macro- and micronutrient intakes.

\section{Dietary patterns and depression}

Multivariable-adjusted odds ratios for depression across quintile categories of dietary pattern scores are provided in Table 5. There was no significant relationship between the traditional, Western and fast food dietary patterns and odds of depression after taking all potential confounders into account. However, individuals in the third quintile of the lacto-vegetarian dietary pattern tended to have lower odds of depression ( $\mathrm{OR}=0.80 ; 95 \% \mathrm{CI} 0.61,1.05)$. When the analysis was done stratified by sex, we found no associations between the fast food and vegetarian dietary patterns and depression in men. However, men in the second quintile of the traditional dietary pattern had $41 \%$ lower odds for depression compared with those in the first quintile ( $\mathrm{OR}=0.56 ; 95 \% \mathrm{CI} 0.35,0.89, P<0.05)$. Also, individuals in the third quintile tended to have $37 \%$ lower odds for depression (OR $=0.63$; 95\% CI 0.40, 1.00, $P<0.05)$. Men in the third quintile of the Western dietary pattern had $73 \%$ higher chance for depression $(\mathrm{OR}=1.73$; $95 \%$ CI 1.07, 2.81, $P<0 \cdot 05$ ).

Among women, we did not find any significant association between the fast food dietary pattern and depression. However, the traditional dietary pattern was associated with greater odds of depression (OR $=1.42$; $95 \%$ CI 1.01, $1.99, P<0.05)$. After adjustment for age, women in the third quintile of the lacto-vegetarian dietary pattern had $35 \%$ lower odds for depression (OR $=0.65 ; 95 \%$ CI $0.48,0.87$, $P<0.05)$ than those in the first quintile. This association remained significant even after further adjustments for confounders. No significant associations were seen between major dietary patterns and psychological disorders in normal-weight or overweight participants. 


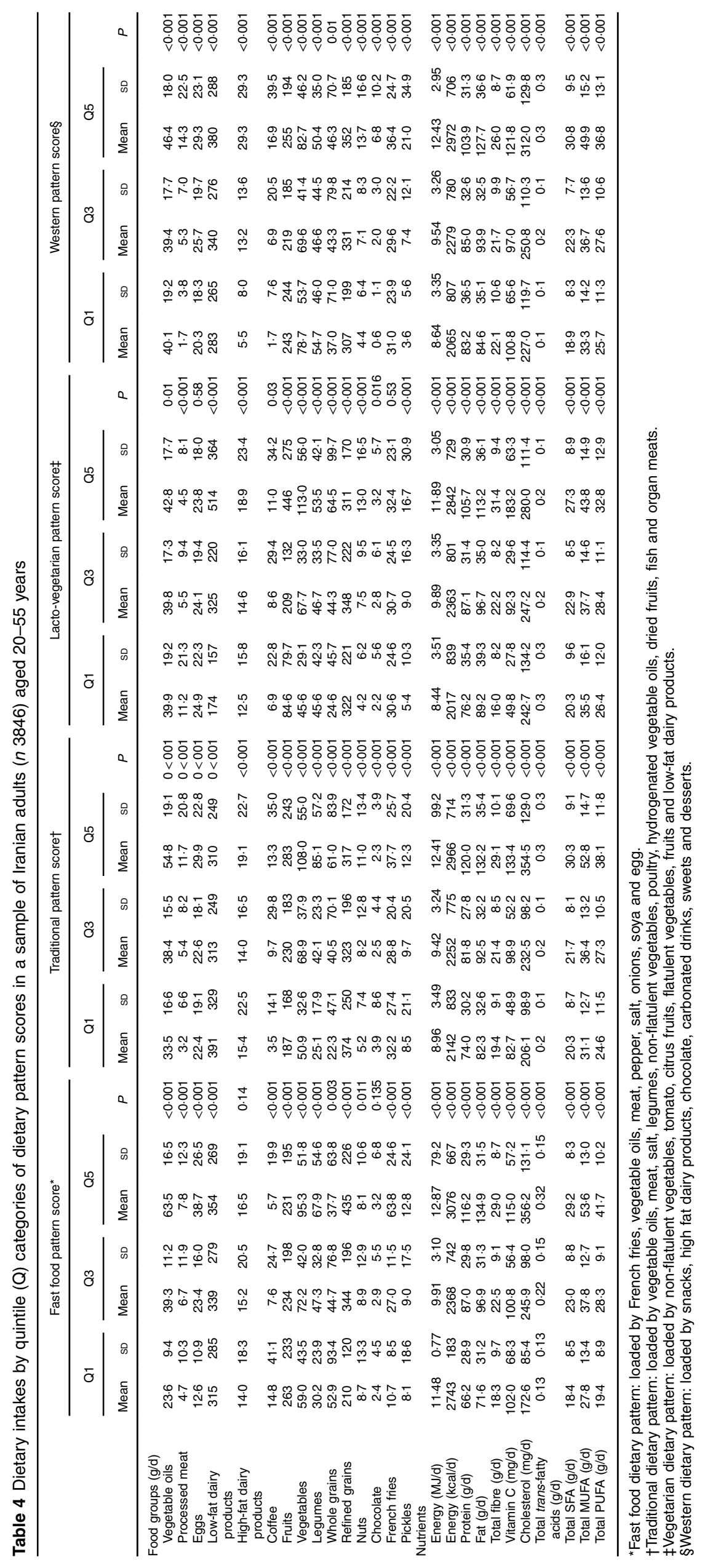




\section{Dietary patterns and anxiety}

Multivariable-adjusted odds ratios for anxiety across quintile categories of dietary pattern scores are provided in Table 6. No significant associations were observed between major dietary patterns and anxiety.

When the analysis was stratified by sex, we observed no link between major dietary patterns and anxiety in men. However, women in the third $(\mathrm{OR}=1.56$; $95 \%$ CI 1.00 , $2.42, P<0.05)$ and fourth quintiles $(\mathrm{OR}=1.57 ; 95 \% \mathrm{CI}$ $1 \cdot 01,2 \cdot 41, P<0.05)$ of the traditional dietary pattern tended to be more anxious than those in the lowest quintile. There were no significant relationships between other dietary patterns and anxiety among women. Stratified analysis by BMI status revealed no significant relationships between major dietary patterns and anxiety in the overweight participants. However, in normal-weight participants, those in the third quintile of the traditional dietary pattern had greater odds for anxiety than those in the first quintile $(\mathrm{OR}=1.89$; $95 \% \mathrm{CI} 1.12$, 3.08, $P<0.05)$. Furthermore, those in the second and third quintiles of the lactovegetarian dietary pattern were less likely to have anxiety. The Western dietary pattern was also associated with increased odds of anxiety in normal-weight participants $(\mathrm{OR}=2 \cdot 05 ; 95 \%$ CI $1 \cdot 22,3 \cdot 46, P<0 \cdot 05)$.

\section{Dietary patterns and psychological distress}

Multivariable-adjusted odds ratios for psychological distress across quintile categories of dietary pattern scores are provided in Table 7 . There were no significant associations between the traditional and Western dietary patterns and psychological distress in the whole population. However, those in the third $(\mathrm{OR}=0.77 ; 95 \%$ CI $0.57,1.01)$ and top quintiles (OR $=0.79 ; 95 \%$ CI 0.59 , 1.02) of the lactovegetarian dietary pattern tended to have lower odds for psychological distress compared with those in the lowest quintile. In our stratified analysis by sex, there was a significant increasing trend in the odds of psychological distress across increasing quintiles of the fast food dietary pattern among women ( $P$ trend=0.02); however, no significant associations were seen in men. Furthermore, our stratified analysis by BMI status revealed no significant associations between major dietary patterns and psychological distress in normal-weight and overweight participants.

Mean scores on the psychological disorders across quintiles of dietary pattern scores are provided in Table 8. There were no significant relationships between mean scores of dietary patterns and psychological disorders.

\section{Discussion}

In the current cross-sectional study exploring the relationship between dietary patterns and psychological disorders in the Iranian adult population, we observed that the lactovegetarian dietary pattern was protectively associated with depression in women and tended to be associated with 
decreased odds of anxiety in normal-weight participants. In addition, the traditional dietary pattern was inversely associated with depression in men, but with increased odds of depression in women and greater odds of anxiety in normalweight participants. The Western dietary pattern was also associated with increased odds of depression in men and greater odds of anxiety in normal-weight participants. Also the lacto-vegetarian dietary pattern tended to be associated with decreased odds of psychological distress. Furthermore, the fast food dietary pattern was protectively associated with psychological distress in women. These associations persisted in multivariate models adjusting for a wide range of potential confounding variables. To the best of our knowledge, the present study is among the first investigations examining the associations between major dietary patterns and anxiety and psychological distress in a Middle Eastern country.

Depression is one of the most prevalent psychological disorders in the world ${ }^{(8)}$, especially in developing countries ${ }^{(21,22)}$. We found no significant associations between major dietary patterns and depression in the whole population; however, sex-stratified analyses revealed a protective association between the traditional dietary pattern and depression in men. Furthermore, among women, the lactovegetarian dietary pattern was inversely associated with depression. Also the Western dietary pattern was associated with increased odds of depression in men. These findings are in line with previous reports that documented a significant association between major dietary patterns and depression. Earlier studies have mostly been conducted in Western nations. For instance, in a population of 1046 Australian adult women, a traditional dietary pattern that included high intakes of vegetables, fruit, beef, lamb, fish and wholegrain foods was associated with a lower likelihood of depressive symptoms $^{(16)}$. However, their findings must be interpreted cautiously because Jacka et $a l .{ }^{(16)}$ did not control for energy intake in their analyses. Although they obtained energyadjusted scores of dietary patterns in their study, the confounding effect of energy intake on depression was not controlled for. Furthermore, the ingredients of their traditional dietary pattern were different from ours. Moreover, while we applied a validated HADS questionnaire to identify depressed persons, Jacka et al. used the DSM-IV (Diagnostic and Statistic Manual of Mental Disorders, 4th edition) for this objective. Earlier studies have shown that the HADS questionnaire has a limited ability to identify major depression compared with a clinical interview measuring tool ${ }^{(29)}$. Another report in men also found an inverse relationship between a traditional Norwegian dietary pattern and risk of depression; however, they failed to find a significant association in women ${ }^{(18)}$. Findings of a cross-sectional study in middle-aged British women indicated a 'processed food' dietary pattern as increasing the risk and a 'whole food' dietary pattern as protective against depression ${ }^{(17)}$. Different study designs, lack of control for several confounders as well as different tools used for the assessment of depressive symptoms might explain the discrepant findings. 


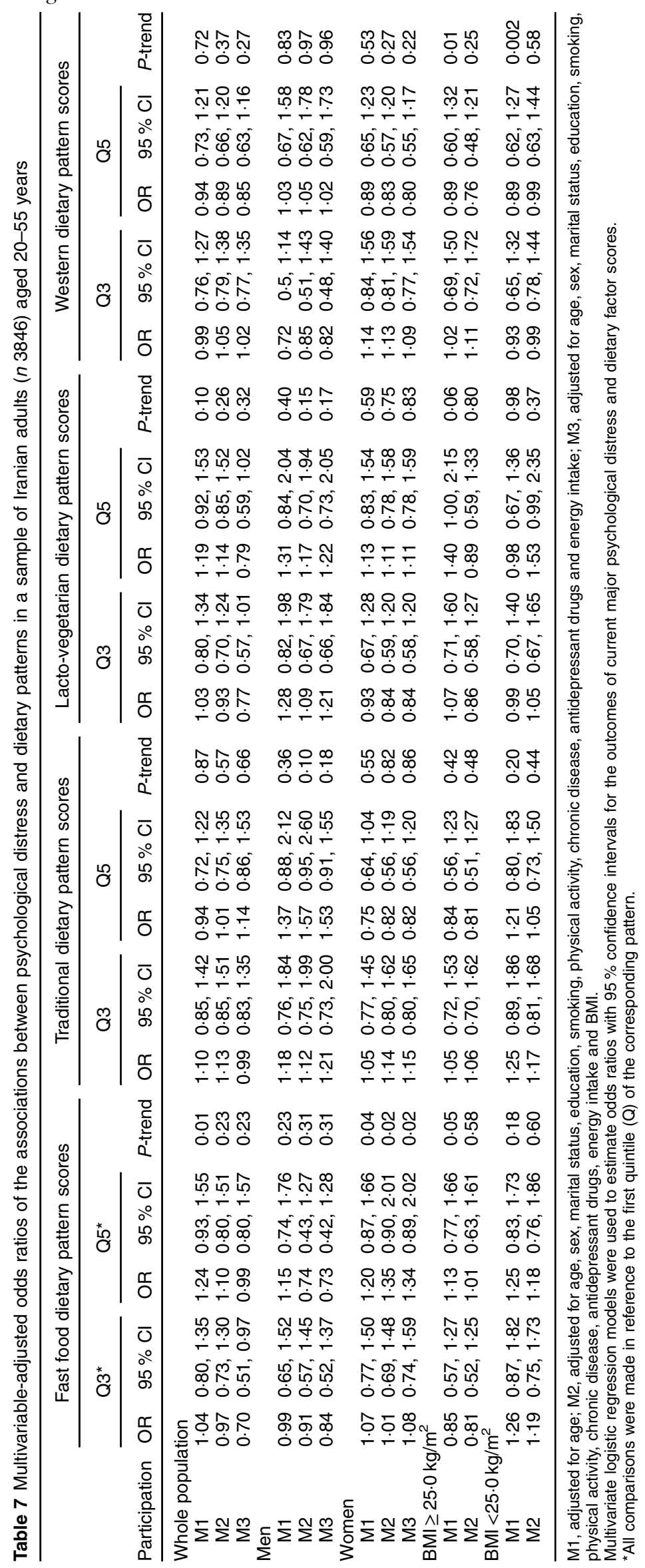




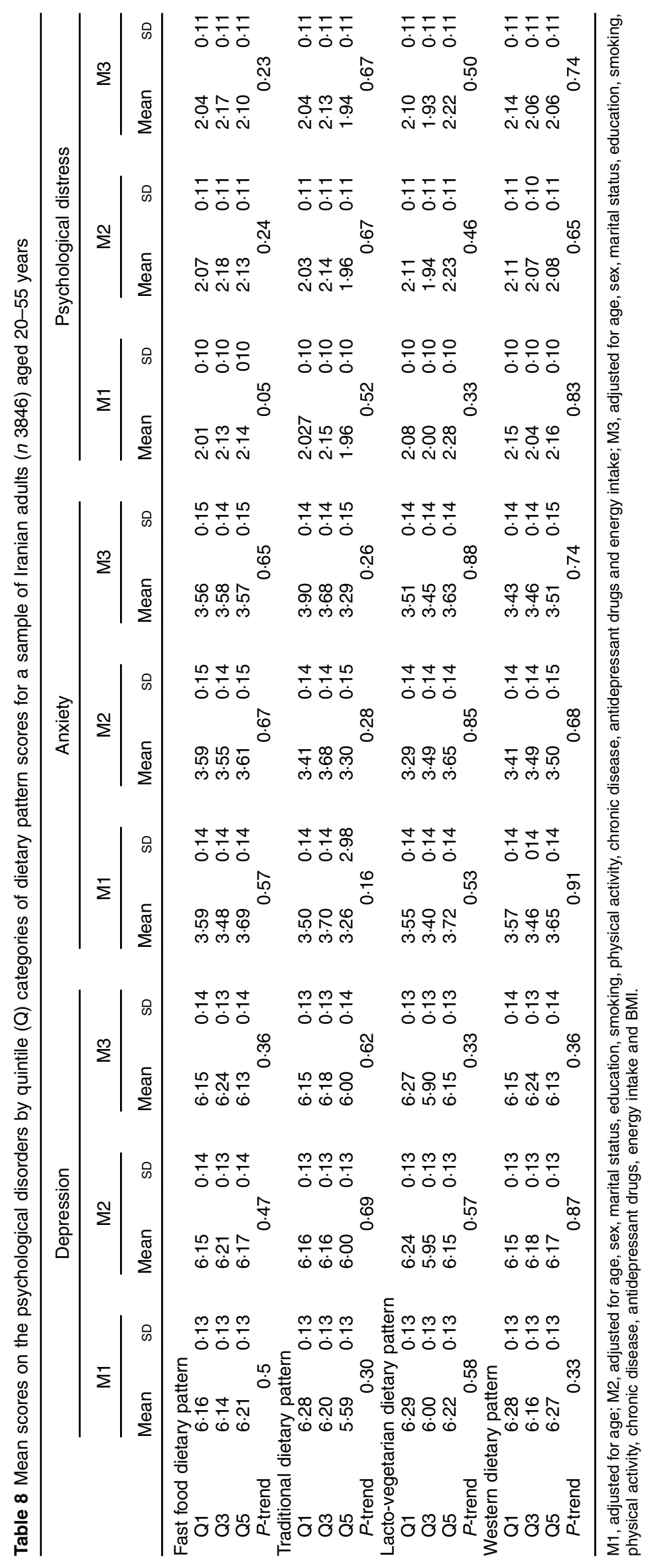


We found that the traditional dietary pattern was associated with increased odds of anxiety in women and normal-weight participants. The Western dietary pattern was associated with increased odds and the lactovegetarian diet with decreased odds of anxiety in normal-weight participants. In contrast to our findings, Jacka et al. ${ }^{(18)}$ found a protective association between their traditional dietary pattern and anxiety in women, but not in men. Another study in Australian women ${ }^{(16)}$ reached the same association. The food content of traditional dietary patterns in the different studies, along with the interactions of these foods in the dietary pattern, might provide a reason for the discrepancies.

Similar to our findings, a Western dietary pattern in Norwegian adults was associated with increased odds of anxiety $^{(18)}$. In addition, a prospective study reached a significant direct association between adherence to the Western dietary pattern and anxiety ${ }^{(32)}$.

We did not observe significant associations between our major dietary patterns and psychological distress. This is in contrast to the findings of Jacka et al. ${ }^{(16)}$, who reported a significant positive association between their Western dietary pattern and this condition. They did not find any significant association between their traditional and modern dietary patterns and psychological distress. Lack of adjustment for total energy intake in the study of Jacka et $a l .{ }^{(16)}$ might provide the reason for this discrepancy. Furthermore, the difference in food content of the major dietary patterns between the two studies might also contribute to this difference.

The exact mechanisms through which dietary intakes might affect psychological disorders are unknown. Psychological disorders, in particular depression, are the result of interaction between genetic, hormonal, immunological, biochemical and neurodegenerative factors. Dietary factors might influence mental function through affecting inflammatory processes and modulating oxidative stress. Earlier studies have shown that elevated inflammation plays a key role in psychological disorders ${ }^{(33)}$. Plant-based foods loaded on our lacto-vegetarian dietary pattern are rich in bioactive compounds being potentially associated with decreased inflammation. Consumption of fruits and vegetables, which include high amounts of folate and antioxidants, has been associated with decreased inflammation and oxidative stress in adults ${ }^{(11)}$. Therefore, the protective association of the lacto-vegetarian dietary pattern with psychological disorders might be explained by its high content of antioxidants ${ }^{(10)}$. Legumes and wholegrain cereals that were loaded on our traditional dietary pattern contain higher amounts of folate and other B vitamins. These nutrients might have favourable effects on psychological conditions through reducing serum homocysteine levels as well as through synthesis of monoamines including dopamine and serotonin in the brain $^{(12)}$. Increased concentrations of serum homocysteine and decreased levels of dopamine and serotonin have been associated with increased risk of depressive disorders ${ }^{(10-12)}$.
Folate and B vitamins are cofactors in the methylation process of homocysteine to methionine. Methionine plays a key role in the production of monoamine transmitters ${ }^{(12,34)}$.

It seems that high intakes of fish (which contain longchain $n$-3 PUFA) in the traditional dietary pattern can also help brain function. This might also contribute to the favourable inverse association of the traditional dietary pattern and anxiety in normal-weight participants. The Western dietary pattern is composed of refined carbohydrates which could be related with elevated C-reactive protein $^{(33)}$. Consumption of dietary factors in the Western dietary pattern has also been associated with decreased brain-derived neurotrophic factor levels ${ }^{(35)}$. This factor has been shown to protect neurons from oxidative stress and induces neurogenesis in the short term ${ }^{(36)}$.

It seems that the associations of dietary patterns with psychological disorders are sex dependent. In a systematic review, this sex difference has been highlighted ${ }^{(11)}$. Biases in dietary reporting between men and women might explain this difference by sex. In addition, gonadal hormones might lead to differential responses to possible mediating factors ${ }^{(11)}$.

The present study has several strengths. To the best of our knowledge, it is the first study that reports the association between major dietary patterns and psychological disorders in a Middle Eastern country. Furthermore, we controlled for a wide range of confounders that might affect psychological conditions. In addition, the large sample size of the study including both sexes must also be kept in mind. Some limitations should also be taken into account in the interpretation of our findings. First of all, due to the cross-sectional design of the study, we cannot confer causality. Some mental disorders such as depression or anxiety might lead to changes in appetite and dietary intakes of the participants; therefore, poor diet quality may be the result of mental health symptoms, rather than a causative factor. We used factor analysis to identify dietary patterns. This method includes several subjective decisions, such as the consolidation of food items into food groups, the number of factors extracted, the method of rotation and labelling of the factors. As the study participants were adults working in health centres, selection bias is a concern that could in turn influence the findings. However, participants were selected from different socio-economic categories throughout the Isfahan province and the studied population covered a wide range of socio-economic categories from both urban and rural areas. Despite these efforts, selection bias is a limitation in the current study and the findings cannot easily be generalized to the general population of Iranian adults. Another potential limitation is measurement error, which is a recognized feature of any dietary assessment method. Due to the use of an FFQ, misclassification of study participants is another concern. Furthermore we cannot exclude the possibility of residual confounding in the analysis due to unmeasured or imprecisely measured factors. 


\section{Conclusion}

In conclusion, we found some significant associations between major dietary patterns and psychological disorders. The implications of the current findings to the general population are to recommend increased intakes of fruits, citrus fruits, vegetables, tomatoes and low-fat dairy products to reduce the chance of psychological disorders. In addition, the general public should be aware to reduce consumption of the Western dietary pattern that was characterized by high intakes of snacks, high-fat dairy products, chocolate, carbonated drinks, sweets and desserts, to protect them against these disorders.

\section{Acknowledgements}

Financial support: The study was financially supported by the Deputy for Research, Isfahan University of Medical Sciences (IUMS) jointly with a grant for a PhD student from Tehran University of Medical Sciences (TUMS), Tehran, Iran. The funders had no role in the design, analysis or writing of this article. Conflict of interest: None. Authorship: M.H., M.V. and A.E. contributed in conception, design and drafting of the manuscript. M.H., A.E., A.F. and R.M. contributed in data analysis. H.A., A.H.K. and P.A. contributed in data collection and manuscript drafting. All authors read and approved the final manuscript. Ethics of buman subject participation: The IUMS Ethics Committee as well as the TUMS Ethics Committee reviewed the study aims and procedures and then approved the study ethically for conduct.

\section{References}

1. Hu FB (2002) Dietary pattern analysis: a new direction in nutritional epidemiology. Curr Opin Lipidol 13, 3-9.

2. Huijbregts P, Feskens E, Räsänen L et al. (1997) Dietary pattern and 20 year mortality in elderly men in Finland, Italy, and The Netherlands: longitudinal cohort study. BMJ 315, 13-17.

3. Kant AK, Graubard BI \& Schatzkin A (2004) Dietary patterns predict mortality in a national cohort: the National Health Interview Surveys, 1987 and 1992. J Nutr 134, 1793-1799.

4. Le Port A, Gueguen A, Kesse-Guyot E et al. (2012) Association between dietary patterns and depressive symptoms over time: a 10-year follow-up study of the GAZEL cohort. PLoS One 7, e51593.

5. Reedy J, Wirfält E, Flood A et al. (2010) Comparing 3 dietary pattern methods - cluster analysis, factor analysis, and index analysis - with colorectal cancer risk: the NIH-AARP Diet and Health Study. Am J Epidemiol 171, 479-487.

6. Kessler RC, Chiu WT, Demler O et al. (2005) Prevalence, severity, and comorbidity of 12-month DSM-IV disorders in the National Comorbidity Survey Replication. Arch Gen Psychiatry 62, 617-627.

7. Kessler RC, Berglund P, Demler O et al. (2005) Lifetime prevalence and age-of-onset distributions of DSM-IV disorders in the National Comorbidity Survey Replication. Arch Gen Psychiatry 62, 593-602.
8. Worley H (2006) Depression a Leading Contributor to Global Burden of Disease. Washington, DC: Population Reference Bureau.

9. Sánchez-Villegas A, Verberne L, De Irala J et al. (2011) Dietary fat intake and the risk of depression: the SUN Project. PLoS One 6, e16268.

10. Murakami K, Miyake Y, Sasaki S et al. (2010) Dietary folate, riboflavin, vitamin B-6, and vitamin B-12 and depressive symptoms in early adolescence: the Ryukyus Child Health Study. Psychosom Med 72, 763-768.

11. Murakami K \& Sasaki S (2009) Dietary intake and depressive symptoms: a systematic review of observational studies. Mol Nutr Food Res 54, 471-488.

12. Kamphuis MH, Geerlings MI, Grobbee DE et al. (2008) Dietary intake of B6-9-12 vitamins, serum homocysteine levels and their association with depressive symptoms: the Zutphen Elderly Study. Eur J Clin Nutr 62, 939-945.

13. Appleton KM, Rogers PJ \& Ness AR (2010) Updated systematic review and meta-analysis of the effects of $n-3$ long-chain polyunsaturated fatty acids on depressed mood. Am J Clin Nutr 91, 757-770.

14. Hibbeln JR (1998) Fish consumption and major depression. Lancet 351, 1213.

15. Calon F (2011) Omega-3 polyunsaturated fatty acids in Alzheimers disease: key questions and partial answers. Curr Alzheimer Res 8, 470-478.

16. Jacka FN, Pasco JA, Mykletun A et al. (2010) Association of Western and traditional diets with depression and anxiety in women. Am J Psychiatry 167, 305-311.

17. Akbaraly TN, Brunner EJ, Ferrie JE et al. (2009) Dietary pattern and depressive symptoms in middle age. $\mathrm{Br} J$ Psychiatry 195, 408-413.

18. Jacka FN, Mykletun A, Berk M et al. (2011) The association between habitual diet quality and the common mental disorders in community-dwelling adults: the Hordaland Health study. Psychosom Med 73, 483-490.

19. Weng TT, Hao JH, Qian QW et al. (2012) Is there any relationship between dietary patterns and depression and anxiety in Chinese adolescents? Public Health Nutr 15, 673-682.

20. Esmaillzadeh A \& Azadbakht L (2008) Major dietary patterns in relation to general obesity and central adiposity among Iranian women. J Nutr 138, 358-363.

21. Mohammadi MR, Davidian H, Noorbala AA et al. (2005) An epidemiological survey of psychiatric disorders in Iran. Clin Pract Epidemiol Ment Health 1, 16.

22. Shen YC, Zhang MY, Huang YQ et al. (2006) Twelve-month prevalence, severity, and unmet need for treatment of mental disorders in metropolitan China. Psychol Med 36, 257-267.

23. Kawakami N, Takeshima T, Ono Y et al. (2005) Twelvemonth prevalence, severity, and treatment of common mental disorders in communities in Japan: preliminary finding from the World Mental Health Japan Survey 2002-2003. Psychiatry Clin Neurosci 59, 441-452.

24. Kessler RC1, Berglund P, Demler O et al. (2003) The epidemiology of major depressive disorder: results from the National Co-morbidity Survey Replication (NCS-R). JAMA 289, 3095-3105.

25. Esmaillzadeh A \& Azadbakht L (2008) Food intake patterns may explain the high prevalence of cardiovascular risk factors among Iranian women. J Nutr 138, 1469-1475.

26. Jahromi SR, Toghae M, Jahromi MJ et al. (2012) Dietary pattern and risk of multiple sclerosis. Iran J Neurol 11, 47-53.

27. Adibi P, Ammar AH, Esmaillzadeh A et al. (2012) The study on the epidemiology of psychological, alimentary health and nutrition (SEPAHAN): overview of methodology. $J$ Res Med Sci 17, 291-297. 
28. Keshteli A, Esmaillzadeh A, Rajaie S et al. (2014) A dishbased semi-quantitative food frequency questionnaire for assessment of dietary intakes in epidemiologic studies in Iran: design and development. Int J Prev Med 5, 29-36.

29. Montazeri A, Vahdaninia M, Ebrahimi M et al. (2003) The Hospital Anxiety and Depression Scale (HADS): translation and validation study of the Iranian version. Health Qual Life Outcomes 1, 14.

30. Montazeri A, Harirchi AM, Shariati M et al. (2003) The 12-item General Health Questionnaire (GHQ-12): translation and validation study of the Iranian version. Health Qual Life Outcomes 1, 66.

31. National Health Service (2006) The General Practice Physical Activity Questionnaire (GPPAQ). London: Department of Health.

32. Chocano-Bedoya PO, O'Reilly EJ, Lucas M et al. (2013) Prospective study on long-term dietary patterns and incident depression in middle-aged and older women. $\mathrm{AmJ}$ Clin Nutr 98, 813-820.

33. Maes M (1999) Major depression and activation of the inflammatory response system. In Cytokines, Stress and Depression, pp. 25-46 [R Dantzer, EE Wollmann and R Yirmiya, editors]. New York: Springer.

34. Crider KS, Yang TP, Berry RJ et al. (2012) Folate and DNA methylation: a review of molecular mechanisms and the evidence for folate's role. Adv Nutr 3, 21-38.

35. Molteni R, Barnard RJ, Ying Z et al. (2002) A high-fat, refined sugar diet reduces hippocampal brain-derived neurotrophic factor, neuronal plasticity, and learning. Neuroscience 112, 803-814.

36. Shirayama Y, Chen AC, Nakagawa S et al. (2002) Brainderived neurotrophic factor produces antidepressant effects in behavioral models of depression. I Neurosci 22, 3251-3261. 